\title{
Application of frozen thin sectioning immunogold staining to the study of the developing neuroepithelial basal lamina
}

\author{
J.S.T. Rheinheimer and K.S. O'Shea \\ Department of Anatomy and Cell Biology, University of Michigan Medical School, Ann Arbor, MI 48109, USA
}

Received November 7, 1986 / Accepted January 24, 1987

\begin{abstract}
Summary. In order to examine the deposition of basal lamina components in the developing neuroepithelium, a technique for frozen thin sectioning and immunogold staining of early embryonic tissue was developed. Different fixatives and buffer systems were evaluated to determine which best retained immunoreactivity and satisfactory ultrastructure of day 9 and 10 mouse embryos. Fixation in sodium phosphate and sodium bicarbonate buffers did not retain antigenicity, and incubations in TBS (trishydroxymethylaminomethane buffered saline) in an effort to 'restore' immunoreactivity were similarly unsuccessful. Fixation in sodium cacodylate buffer, however, did retain the antigenicity of basal lamina components; the pattern of type IV collagen and laminin distribution was clearly determined. These results represent the first report of on-grid immunocytochemistry of early embryonic material.
\end{abstract}

\section{Introduction}

Cell-cell and cell-extracellular matrix interactions appear to play a significant role in many morphogenetic processes (see review by Hay 1984). To better understand the nature and underlying basis of these interactions, it is critical to determine the time of appearance and pattern of localization of the extracellular matrix material, and to correlate this pattern with the resulting ultrastructural characteristics of a cell or group of cells. Previous attempts to localize cellular and extracellular materials by electron microscopy have relied on pre-embedding immunocytochemical (Mayer et al. 1981; Inoue et al. 1983; Sanders 1984; Herken and Barrach 1985) or histochemical (Mayer et al. 1981; Herken and Barrach 1985; Wilson 1985) techniques, or have been carried out at the light microscope level (Krotoski et al. 1986; Sternberg and Kimber 1986; Tuckett and MorrissKay 1986).

In order to examine the detailed distribution of intraand extracellular components of the developing embryo, as well as to co-localize these highly interactive components, on-grid immunocytochemical studies must be carried out. In the current investigation, the deposition of type IV collagen and laminin in the neuroepithelial basal lamina of day 9 and 10 mouse embryos was studied using frozen thin sectioning (FTS) and immunogold staining (IGS) techniques. Various fixation procedures and buffer systems were evaluated to determine the optimal method for localizing these components in embryos at the transmission electron microscope (TEM) level while retaining satisfactory ultrastructure.

\section{Materials and methods}

Tissue preparation

Male and female CD-1 strain mice (Charles River laboratories) were mated, the morning of finding a vaginal plug considered the first day of gestation. On the 9th or 10th day, pregnant females were killed by cervical dislocation and the embryos removed from the uterus, decidua and chorion.

\section{Fixation}

The embryos, retained in their amnion, were fixed using one of the following procedures:

A) Modified Eldred technique. Embryos were fixed in $4 \%$ paraformaldehyde (Ladd Research Industries, Inc.) and $0.2 \%$ glutaraldehyde (Polysciences, Inc.) in $0.1 \mathrm{M}$ sodium phosphate buffer, $\mathrm{pH} 7.3$, for $1 \mathrm{~h}$, followed by a $12 \mathrm{~h}$ fixation in $4 \%$ paraformalde-

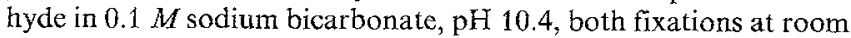
temperature. The embryos were then washed for $1 \mathrm{~h}$ in $0.1 M$ sodium phosphate buffer, pH 7.3, with $0.1 \mathrm{M}$ sucrose (rinse-buffer), followed by a $1 / 2 \mathrm{~h}$ incubation in $1 \%$ sodium borohydride in rinsebuffer. The embryos were again washed in rinse-buffer for approximately 1 h (adapted from Eldred et al. 1983) and immediately infiltrated with sucrose as described below.

B) Phosphate buffer. Embryos were fixed in 4\% paraformaldehyde and $0.2 \%$ glutaraldehyde in $0.1 \mathrm{M}$ sodium phosphate buffer, $\mathrm{pH} 7.3$, for $1 \mathrm{~h}$ at room temperature. The fixative was then replaced with $0.1 \mathrm{M}$ sodium phosphate buffer, pH 7.3 , containing $0.1 \mathrm{M}$ sucrose (rinse-buffer), and the embryos stored at $4^{\circ} \mathrm{C}$ for no more than $20 \mathrm{~h}$ prior to sucrose infiltration.

C) Phosphate-tris buffer. Embryos were fixed in $4 \%$ paraformaldehyde and $0.2 \%$ glutaraldehyde in $0.1 M$ sodium phosphate buffer, $\mathrm{pH} 7.3$, for $1 \mathrm{~h}$ at room temperature. The tissue was washed in $0.1 M$ trishydroxymethylaminomethane (Sigma Chemical Co.) buffered saline (TBS-buffer), $\mathrm{pH} 7.3$, for $13^{1 / 2} \mathrm{~h}$ at $4^{\circ} \mathrm{C}$ (adapted from Herken and Barrach 1985), then immediately infiltrated with sucrose in either $0.1 M$ phosphate buffer or $0.1 M$ TBS-buffer as described below.

D) Tris buffer. Embryos were fixed in 4\% paraformaldehyde and $0.2 \%$ glutaraldehyde in $0.1 M$ TBS-buffer, $\mathrm{pH} 7.3$, for $1 \mathrm{~h}$ at room temperature. The tissue was washed in $0.1 M$ TBS-buffer for $13^{1 / 2} \mathrm{~h}$ at $4^{\circ} \mathrm{C}$ (adapted from Herken and Barrach 1985), followed 
by immediate infiltration with sucrose in $0.1 \mathrm{M}$ TBS-buffer, as described below.

E) Cacodylate buffer. Embryos were fixed in $4 \%$ paraformaldehyde and $0.2 \%$ glutaraldehyde in $0.100 \mathrm{M}, 0.075 \mathrm{M}$, or $0.050 \mathrm{M}$

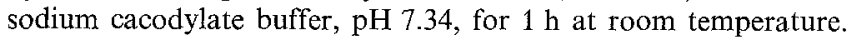
The fixative was then replaced with the appropriate rinse-buffer. The embryos were stored at $4^{\circ} \mathrm{C}$ for no more than $20 \mathrm{~h}$ prior to sucrose infiltration.

In some experiments, various concentrations of glutaraldehyde $(0.2 \%-1.0 \%)$ were used.

\section{Cryoprotection}

Over a period of approximately $4 \mathrm{~h}$, at room temperature, the embryos were gradually infiltrated with increasing concentrations of sucrose (in the appropriate rinse-buffer) until $2.3 \mathrm{M}$ sucrose was reached (Tokuyasu 1973). During infiltration, the embryos were rotated on a Pelco Infiltron.

The embryos were then dissected into $0.5 \mathrm{~mm}$ blocks so that each tissue block included neuroepithelium. Each piece of tissue was mounted on a copper chuck, oriented for transverse sectioning, and frozen by immersion in liquid nitrogen. In some experiments, Tissue-Tek Optimal Cutting Temperature Compound (OCT; Miles Scientific) was used to mount the tissue block on the chuck before freezing.

\section{Cryosectioning}

Ultrathin cryosections were cut using a Sorvall MT-2 Ultramicrotome fitted with the Dupont/Sorvall FTS System (Christensen 1971). The sections were picked up by the loop method (using $2.3 M$ sucrose in the appropriate rinse-buffer) and mounted on 200-mesh nickel grids that had been previously coated with both formvar $(0.5 \%)$ and carbon (moderate to heavy coating), and deionized for $60 \mathrm{~s}$ in a glow-discharge chamber. In some experiments, saponin $(0.010 \%, 0.005 \%$ or $0.001 \%)$ was added to the sucrose solution used to transfer sections from the knife to the grid. The grids were immediately floated on the appropriate rinsebuffer to remove the sucrose from the tissue sections.

\section{Immunocytochemistry}

Briefly, the following immunocytochemical procedure was used. Unless otherwise noted, all dilutions were made with the appropriate buffer.

The grids were first washed in $0.01 M$ glycine, then treated with $0.1 \%$ sodium borohydride (unless the tissue had been incu- bated en bloc with sodium borohydride). The grids were washed, treated with normal goat serum (NGS) diluted 1:20, and incubated for $2 \mathrm{~h}$ in either anti-type IV collagen (supplied by Dr. H. Furthmayr, Yale University) diluted 1:20, or anti-laminin (kindly donated by Dr. J.P. McCoy, University of Michigan) diluted 1:25 or 1:50. Antigens were obtained from EHS tumor; both antibodies were affinity purified and exhibited no cross-reactivity. Controls were incubated in either the appropriate buffer or NGS in place of the primary antibody. All grids were washed, then incubated for $30 \mathrm{~min}$ in the secondary antibody and gold marker, goat antirabbit (IgG) colloidal gold (15 nm particles) conjugate (Janssen Pharmaceuticals, distributed by Structure Probe, Inc.) diluted 1:5. The tissue was fixed in $2 \%$ glutaraldehyde and stained with $1 \%$ osmium tetroxide, followed by $0.5 \%$ uranyl acetate in $0.05 \mathrm{M}$ sodium hydrogen maleate buffer. Following stepwise dehydration, the grids were postembedded in Medium Grade L.R. White Acrylic Resin (The London Resin Co., Ltd., distributed by Structure Probe, Inc.) and cured in a $65^{\circ} \mathrm{C}$ oven overnight (adapted from Keller et al. 1984). The sections were examined and photographed on a Philips 400 transmission electron microscope.

\section{Results}

The neuroepithelium and its subjacent basal lamina were examined in all experiments.

\section{A. Modified Eldred technique}

In embryos pre-fixed in paraformaldehyde and glutaraldehyde, fixed in paraformaldehyde, and incubated en bloc in sodium borohydride before sucrose infiltration, the neuroepithelium was fully intact. Tightly packed pseudostratified neuroepithelial cells extended from the apical lumen to the basal lamina, with tight junctions located at their apices (Fig. 1). Mitochondria, Golgi apparatus, and plasmalemmae were well-defined although in some samples, nuclear membranes of the interphase nuclei were scalloped and small spaces in the cytoplasmic ground substance were observed. The neuroepithelial cells were bounded by a basal lamina composed of a lamina lucida, lamina densa, and an outer layer containing collagen fibrils. Laminae lucida and densa were sometimes well-defined but more typically, these two layers could not be distinguished and occasionally were totally absent. In regions where the basal lamina was preserved, almost no localization of laminin or type IV collagen by colloidal gold particles was observed (Fig. 2).

Figs. 1-6. Transverse frozen thin sections through the neuroepithelium of day 10 embryos, illustrating the effects of various fixation and buffer systems on neuroepithelial morphology (Figs. 1 and 3) and on the immunoreactivity of its basal lamina (Figs. 2, 4, 5, 6)

Fig. 1. Low magnification view illustrating that neuroepithelial cells in an embryo processed by the modified Eldred technique were typically tightly packed, with well-defined plasmalemmae and organelles. Nuclear membranes were often scalloped. Scale bar $=2 \mu \mathrm{m}$

Fig. 2. At higher magnification, little basal lamina Iocalization of laminin was observed in embryos processed by the modified Eldred technique. Anti-laminin dilution 1:25. Scale bar $=0.5 \mu \mathrm{m}$

Fig. 3. In embryos processed in phosphate buffer, gaps between neuroepithelial cells, and intracellular spaces, were present. Scale bar $=2 \mu \mathrm{m}$

Fig. 4. There was little laminin localization in the basal lamina of embryos processed in phosphate buffer. Anti-laminin dilution $1: 50$. Scale bar $=0.5 \mu \mathrm{m}$

Fig. 5. In embryos processed in phosphate-tris buffer, specific labeling of laminin in the basal lamina could not be distinguished from the high background. Anti-laminin dilution 1:25. Scale bar $=0.5 \mu \mathrm{m}$

Fig. 6. The pattern of laminin distribution in the basal lamina was well-marked in embryos processed in cacodylate buffer. Antibody dilution $1: 25$. Scale bar $=0.5 \mu \mathrm{m}$ 

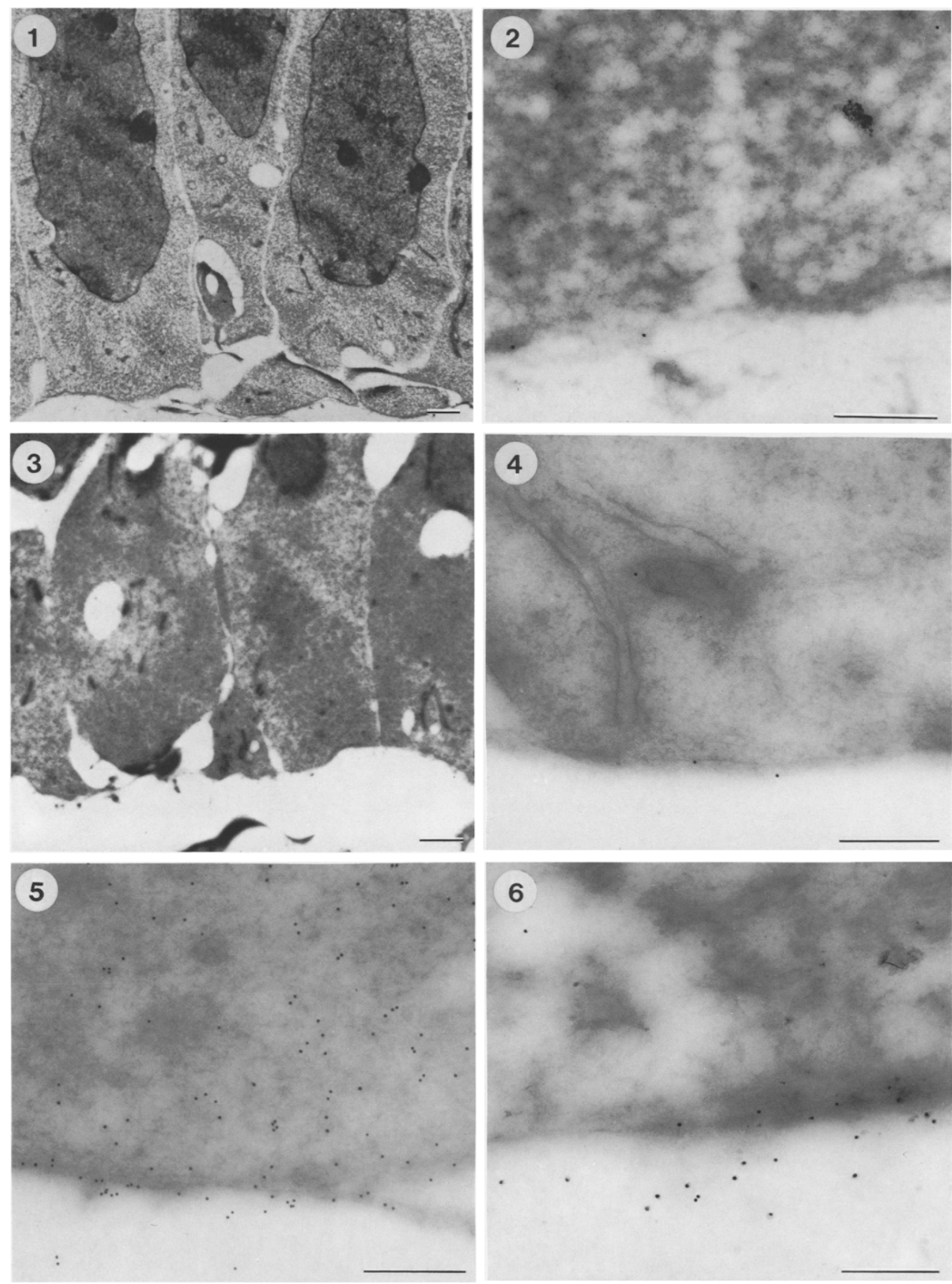

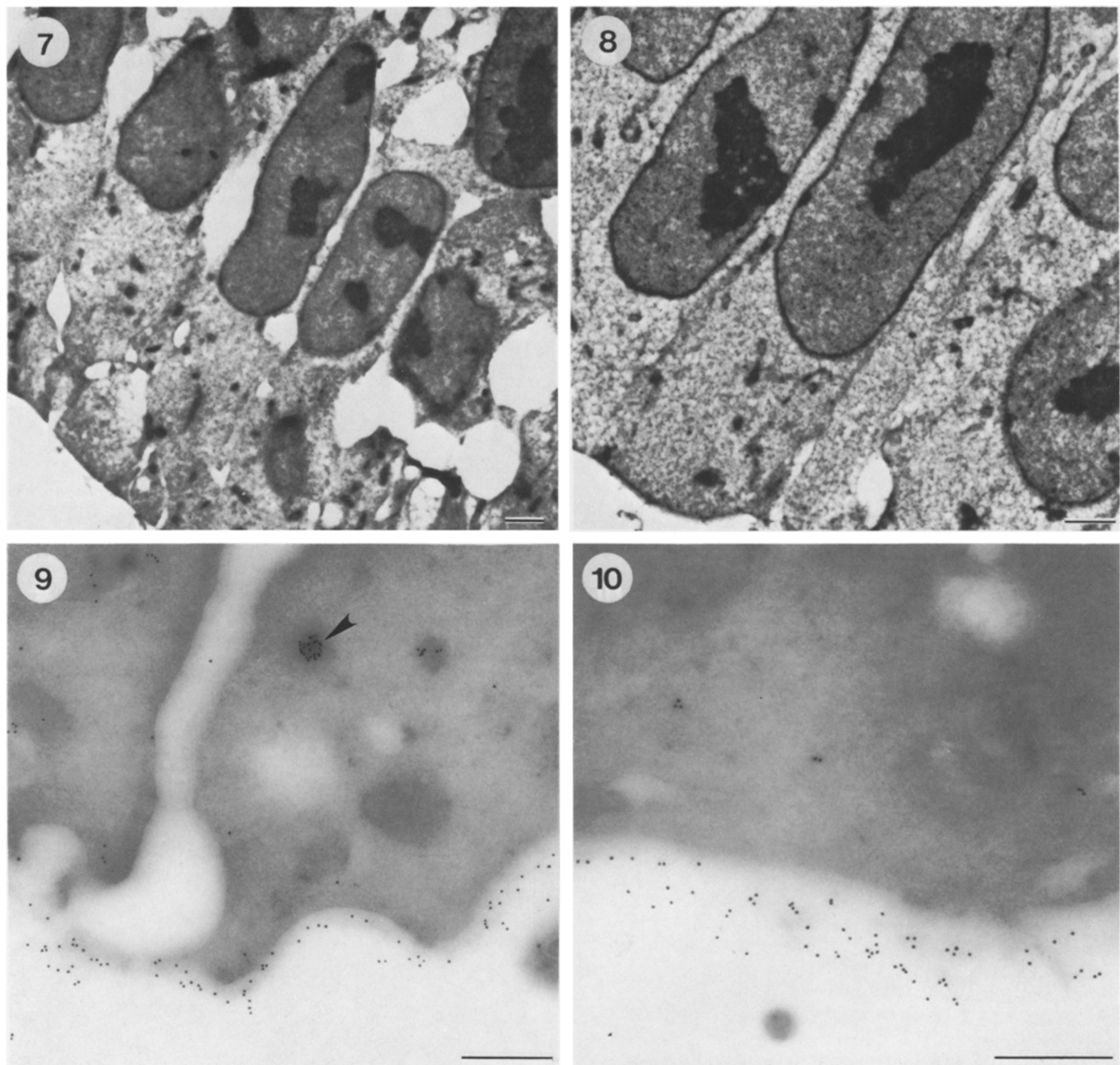

Figs. 7-10. Transverse frozen thin sections of embryos processed in sodium cacodylate buffer, showing both the range in tissue quality obtained and type IV collagen localization

Fig. 7. In day 10 embryos, tissue quality varied, with some regions of the neuroepithelium containing greater amounts of inter- and intracellular space. Scale bar $=2 \mu \mathrm{m}$

Fig. 8. Other areas of the neuroepithelium in day 10 embryos were comprised of tightly packed cells; nuclear membranes were smooth and unbroken. Scale bar $=2 \mu \mathrm{m}$

Fig. 9. The neuroepithelial basal lamina was typically continuous in day 9 embryos, with considerable deposition of type IV collagen. Prior to its extrusion from the cell, precursor to type IV collagen was also localized (arrow) in vesicles within the neuroepithelium. Anti-type IV collagen dilution 1:20. Scale bar $=0.5 \mu \mathrm{m}$

Fig. 10. At higher magnification, the pattern of type IV collagen distribution clearly followed the contour of the basal lamina in day 9 embryos. Antibody dilution 1:20. Scale bar $=0.5 \mu \mathrm{m}$

\section{B. Phosphate buffer}

The neuroepithelium also was intact in tissue fixed in $4 \%$ paraformaldehyde and $0.2 \%$ glutaraldehyde in $0.1 \mathrm{M}$ sodium phosphate buffer. Tight junctions held the neuroepithe- lial cells together in the apical regions but unlike the tightly adherent cells seen in the previous experiment, large intercellular gaps often occurred in areas in which tight junctions do not form. In addition, spaces in neuroepithelial cell cytoplasm were often observed (Fig. 3). Nuclear membranes 
were unbroken and smooth, and mitochondria and Golgi apparatus were visible. The basal lamina of the neuroepithelium was continuous; however, in most regions, almost no localization of laminin by the colloidal gold particles was observed (Fig. 4).

\section{Phosphate-tris buffer}

The overall morphology of the neuroepithelium was poor in embryos fixed in paraformaldehyde and glutaraldehyde in phosphate buffer, treated with $0.1 M$ TBS-buffer, and infiltrated with sucrose in phosphate buffer. The neuroepithelial cells appeared pulled apart and the cytoplasm largely extracted. Although laminin labeling appeared to follow the contour of the basal lamina, the background was so high that labeling of basal lamina components could not be distinguished from non-specific staining.

When the embryos were infiltrated with sucrose in TBSbuffer rather than in phosphate buffer, the morphology of the neuroepithelium appeared improved although gaps between neuroepithelial cells persisted. Specific labeling of basal lamina components could not be distinguished from background (Fig. 5).

\section{Tris buffer}

In the embryos fixed, washed for $131 / 2 \mathrm{~h}$, and infiltrated in TBS-buffer, the apical regions of the neuroepithelial cells were adherent but toward their basal portion, they were pulled so far apart that few cells remained attached to the basal lamina. As in the other TBS-buffer experiments, labeling of the basal lamina could not be differentiated from background labeling.

\section{E. Cacodylate buffer}

The neuroepithelium was also intact in embryos fixed in $4 \%$ paraformaldehyde and $0.2 \%$ glutaraldehyde in $0.1 \mathrm{M}$ sodium cacodylate buffer although gaps between neuroepithelial cells in both apical and basal regions as well as holes in the cytoplasm were sometimes present. The nuclear membranes were smooth; Golgi apparatus and mitochondria were visible. The neuroepithelial basal lamina was largely intact and laminin was heavily labeled by colloidal gold particles (Fig. 6). Localization along the basal lamina was easily distinguished from the light background.

In embryos fixed in paraformaldehyde and glutaraldehyde in either 0.05 or $0.075 \mathrm{M}$ sodium cacodylate buffer, the neuroepithelium was fully intact. Overall tissue morphology was generally superior to that obtained with $0.1 \mathrm{M}$ sodium cacodylate buffer although tissue quality did vary. Nuclear membranes were smooth and unbroken, and organelles visible. In some regions, gaps between neuroepithelial cells and spaces within the cytoplasm were present (Fig. 7) while in other regions of the same section, the neuroepithelial cells were packed tightly together with few intracellular spaces (Fig. 8). However, the basal lamina of the neuroepithelium was typically continuous with type IV collagen very well localized (Figs. 9 and 10).

\section{Additional observations}

In some experiments, glutaraldehyde concentration was varied, but $0.2 \%$ glutaraldehyde resulted in good structural preservation while best retaining the tissue's antigenicity.
In all controls, no labeling of the basal lamina was observed. An occasional gold particle was seen within the neuroepithelium or in the mesenchymal compartment subjacent to the basal lamina.

In other experiments, saponin was added to the sucrose solution used to transfer frozen thin sections from knife to grid. No differences in ease of section transfer, or in quality of the tissue sections were observed with the addition of saponin.

It was also determined that "gluing" the tissue blocks onto the copper chucks with OCT did not prevent the tissue from loosening from the chuck during storage or sectioning. The tissue blocks did not appear more stable when OCT was used and the additional OCT usually made the block rather large to section.

\section{Discussion}

This study reports the first application of on-grid immunocytochemistry to early embryonic tissue. Due to the difficulty of balancing the requirements of adequate fixation of the highly hydrated embryos with adequate maintenance of tissue immunoreactivity, previous studies have relied on pre-embedding localization techniques for TEM immunocytochemistry. In the current investigation, the FTS and IGS procedures were chosen because frozen thin sections allow the antibody equal access to the surface of the tissue section while avoiding the hindrance of resins during the binding procedure (Keller et al. 1984), and allow the localization of intracellular antigens without permeabilization of cells and resulting loss of ultrastructure.

Choice of fixative is always a vital consideration since different fixation procedures influence both cellular preservation and the antigenicity of the tissue, particularly with embryonic materials (cf., Waterman 1980). In this study, a combination of $4 \%$ paraformaldehyde and $0.2 \%$ glutaraldehyde resulted in satisfactory fixation: the neuroepithelial fine structure was generally well preserved with Golgi apparatus and mitochondrial membranes intact, and with smooth, unbroken nuclear envelopes.

However, the retained immunoreactivity of the neuroepithelial basal lamina varied considerably depending upon which buffer or combination of buffers was used with the fixative. Since sodium phosphate is considered a physiological buffer, and sodium bicarbonate has been reported to better preserve cellular structure than tris or phosphate buffers when used in methacrylate (Hayat 1981), it was not surprising that procedures employing those buffers produced higher quality ultrastructural preservation than cacodylate buffer. But, little to no antigenicity of the neuroepithelial basal lamina components was retained. Immunoreactivity persisted only when sodium cacodylate served as the buffering medium

We have observed similar patterns of immunoreactivity when embryonic tissue was processed for light microscopical localization of type IV collagen and laminin. Embryos were fixed as described above, frozen sections $(8 \mu \mathrm{m})$ cut and processed as above except that goat anti-rabbit IgG was conjugated to fluorescein isothiocyanate (FITC) rather than to colloidal gold. Immunofluorescence was significantly stronger in tissue processed in cacodylate buffer compared to phosphate buffer. The resulting fluorescence in phosphate buffer was often so weak that it could not adequately be recorded photographically. 
The mechanism of action of cacodylate in these results is unclear. While it has previously been suggested that cacodylate may alter membrane permeability, and does better preserve the activity of some enzymes (Hayat 1981), there are no reports of improved immunoreactivity. The nonionic cacodylate buffer may simply stabilize the highly charged molecules which compose the basal lamina and extracellular matrix without altering their conformation. Ions present in buffers may produce conformational changes in these charged molecules, thus rendering portions of the molecule inaccessible to the antibody.

Although localization of laminin and type IV collagen in the neuroepithelial basal lamina was consistent in embryos processed in cacodylate, tissue quality varied. Regions of tightly packed neuroepithelial cells were interspersed with areas containing more inter- and intracellular spaces. Rather than vagaries of fixation, this range in quality may reflect regional variation in tissue morphology at this early stage of development, or may be partially caused by the very nature of frozen thin sectioning. Inherent problems of this technique can be minimized but seldom eliminated. Cryoprotection will reduce ice-crystal damage but the requisite high molarity of sucrose may osmotically shock highly hydrated and delicate embryos. Or, cacodylate itself may produce some of the spaces observed since Wood and Luft (1965) also reported intercellular spaces in tissue processed with cacodylate buffer and embedded in epoxy resin. In the current study, tissue quality did generally meet the requirements of adequate fixation even though - possibly unavoidable - spaces were present. The germane effect of cacodylate on the embryonic neuroepithelium is the retention of the basal lamina's intrinsic antigenicity. The results of this study confirm the light microscopical localization of glycoproteins in the neuroepithelial basal lamina of rat (Tuckett and Morriss-Kay 1986), mouse (Sternberg and Kimber 1986), and chick (Krotoski et al. 1986) embryos.

Other studies of basal laminae at the ultrastructural level have employed tannic acid (Herken and Barrach 1985; Wilson 1985) and ruthenium red (Mayer et al. 1981; Wilson 1985), but these compounds demonstrate only glycosaminoglycan and proteoglycan distribution. Herken and Barrach (1985) attempted to restore basal lamina antigenicity in mouse embryos by using TBS-buffer and pre-embedding techniques but reported no glycoprotein labeling within the embryo.

With the FTS and IGS method, and by processing the tissue with sodium cacodylate buffer, the pattern of type IV collagen and laminin distribution has been determined in the neuroepithelial basal lamina of the mouse embryo on gestational days 9 and 10 and in the developing palate, in day 14 mouse embryos (unpublished). In addition, while the diffuse reaction product of the peroxidase methods (used in pre-embedding localization) can prove difficult to interpret at the ultrastructural level, and ferritin particles are small in size with only moderate electron density, colloidal gold particles are easily resolved and quantified in the electron microscope (Sternberger 1979; Bosman 1983; De Mey 1983). With the FTS and IGS procedures, intracellular labeling is possible, as demonstrated by the localized precursor of type IV collagen in secretory vesicles within the neuroepithelium. In addition, several antigens can be readily co-localized by using different sizes of gold particles.
Current investigations are in progress to localize various additional basal lamina components to determine their time and pattern of deposition, and to quantify that deposition, both prior to and following basal lamina disruption by mutant genes and teratogen exposure.

Acknowledgements. The authors are grateful to Dr. A. Kent Christensen for consultation and to T.K. Komerowski for technical advice during the course of this study. Supported by NIH grant NS-21108.

\section{References}

Bosman FT (1983) Some recent developments in immunocytochemistry. Histochem J 15:189-200

Christensen AK (1971) Frozen thin sections of fresh tissue for electron microscopy, with a description of pancreas and liver. J Cell Biol 51:772-804

De Mey J (1983) A critical review of light and electron microscopic immunocytochemical techniques used in neurobiology. J Neurosci Methods 7:1-18

Eldred WD, Zucker C, Karten HJ, Yazulla S (1983) Comparison of fixation and penetration enhancement techniques for use in ultrastructural immunocytochemistry. J Histochem Cytochem $31: 285-292$

Hay ED (1984) Cell-matrix interaction in the embryo: cell shape, cell surface, cell skeletons, and their role in differentiation. In: Trelstad RL (ed) The role of extracellular matrix in development. Alan R Liss, New York, pp 1-31

Hayat MA (1981) Fixation for electron microscopy. Academic Press, New York, pp 39-48, 353, 364-367

Herken R, Barrach H-J (1985) Ultrastructural localization of type IV collagen and laminin in the seven-day old mouse embryo. Anat Embryol 171:365-371

Inoue S, Leblond CP, Laurie GW (1983) Ultrastructure of Reichert's membrane, a multilayered basement membrane in the parietal wall of the rat yolk sac. J Cell Biol 97:1524-1537

Keller G-A, Tokuyasu KT, Dutton AH, Singer SJ (1984) An improved procedure for immunoelectron microscopy: ultrathin plastic embedding of immunolabeled ultrathin frozen sections. Proc Natl Acad Sci USA 81:5744-5747

Krotoski DM, Domingo C, Bronner-Fraser M (1986) Distribution of a putative cell surface receptor for fibronectin and laminin in the avian embryo. J Cell Biol 103:1061-1071

Mayer BW Jr, Hay ED, Hynes RO (1981) Immunocytochemical localization of fibronectin in embryonic chick trunk and area vasculosa. Dev Biol 82:267-286

Sanders EJ (1984) Labelling of basement membrane constituents in the living chick embryo during gastrulation. J Embryol Exp Morphol 79:113-123

Sternberg J, Kimber SJ (1986) Distribution of fibronectin, laminin and entactin in the environment of migrating neural crest cells in early mouse embryos. J Embryol Exp Morphol 91:267-282

Sternberger LA (1979) Immunocytochemistry. 2nd edn. John Wiley and Sons, New York, p 74

Tokuyasu KT (1973) A technique for ultracryotomy of cell suspensions and tissues. J Cell Biol 57:551-565

Tuckett F, Morriss-Kay GM (1986) The distribution of fibronectin, laminin and entactin in the neurulating rat embryo studied by indirect immunofluorescence. J Embryol Exp Morphol 94:95-112

Waterman RE (1980) Preparation of embryonic tissues for SEM. Scan Electron Microse 2:21-44

Wilson DB (1985) Ultrastructure of the neural basal lamina in loop-tail mice. Acta Anat 123:77-81

Wood RL, Luft JH (1965) The influence of buffer systems on fixation with osmium tetroxide. J Ultrastruct Res 12:22-45 